Bull. Austral. Math. Soc.

VOL. 46 (1992) [187-197]

\title{
A REMARK ON GELFAND DUALITY
}

\author{
Shu-Hao Sun
}

\begin{abstract}
In this paper, we prove a Gelfand-Mulvey type of duality for a certain class of rings which includes the Gelfand rings. We also show that the Maximal Ideal Theorem (MIT) can be replaced by the Prime Ideal Theorem (PIT) in the original Gelfand-Mulvey duality.
\end{abstract}

\section{INTRODUCTION}

The Gelfand duality theorem states that the functor from the category of compact $T_{2}$ spaces to the category of commutative $C^{*}$-algebras, obtained by assigning to each compact $T_{2}$ space $X$ the commutative $C^{*}$-algebra $C(X)$ of continuous complex functions on $X$, determines a duality between these categories. The dual functor is that obtained by assigning to each commutative $C^{*}$-algebra $A$ the compact $T_{2}$ space Max $A$ consisting of the maximal ideals of $A$ endowed with the hull-kernel topology (of which the subsets of $\operatorname{Max} A$ of the form $D(a)=\{M \in \operatorname{Max} A \mid a \notin M\}$ for each $a \in A$ form a basis of the topology). It may be remarked that the maximal ideal space of a commutative ring $A$ is generally neither $T_{2}$ nor functorial on the category of commutative rings. The existence of this functor therefore depends on particular preperties of commutative $C^{*}$-algebras. To extend the Gelfand duality to (not necessarily commutative) rings, Mulvey [4] introduced the following notion: a ring $A$ is called Gelfand if for any two distinct maximal right ideals $M$ and $M^{\prime}$, there exist elements $a \notin M$ and $a^{\prime} \notin M^{\prime}$ such that $a A a^{\prime}=0$. It was shown in [4] that although the definition might appear to be that of a right Gelfand ring, the condition would turn out to be equivalent to that in terms of maximal left ideals and that the maximal ideal space of any Gelfand ring is compact $T_{2}$, and that the assignment to each ring $A$ of the maximal ideal space $\operatorname{Max} A$ is functorial on the category of Gelfand rings and ring homomorphisms. In fact, Mulvey obtained the following:

Received 21st August 1991

The author acknowledges with gratitude the support of the Australia Research Council, and wishes to thank Professor G.M. Kelly for his encouragement and a special thank to Dr K.G. Choo for his assistance.

Copyright Clearance Centre, Inc. Serial-fee code: 0004-9729/92 \$A2.00+0.00. 
THEOREM. [4]. The functor from the category of compact local ringed spaces to the category of Gelfand rings, obtained by assigning to each ringed space $\left(X, O_{X}\right)$ the ring of sections $O_{x}(X)$, determines a duality between these categories.

It may be remarked here that, although Mulvey's definition of Gelfand rings is sensible only in the presence of the maximal ideal Theorem (MIT) - whose validity for all rings is logically equivalent to the Axiom of Choice (AC) - it is possible to give another description of Mulvey's duality by using only the Prime Ideal Theorem (PIT) which is strictly weaker than AC. It is also possible to extend Gelfand-Mulvey's duality to a wider class of rings. These are the main purposes of this paper.

In general, the inverse image of a maximal (right) ideal under a ring homomorphism need not necessarily be a maximal (right) ideal; for rings which are not commutative, the inverse image need not even be a prime ideal. For Gelfand rings, Mulvey has shown that the inverse image of a maximal right ideal determines a unique maximal ideal since each maximal right ideal is completely prime (an ideal $P$ of a ring $R$ is called completely prime if $a b \in P$ implies that $a \in P$ or $b \in P$ ) and the inverse of a completely prime ideal under a ring homomorphism is clearly completely prime. But a maximal ideal is not necessarily completely prime, even for strongly harmonic rings (a ring $R$ is called strongly harmonic if for any two distinct maximal 2-sided ideals $M$ and $M^{\prime}$, there exist elements $a \notin M$ and $a^{\prime} \notin M^{\prime}$ such that $a A a^{\prime}=0$ [2]). However, we shall show that each ring homomorphism $f: R_{1} \rightarrow R_{2}$, where $R_{1}$ and $R_{2}$ are strongly harmonic rings such that each maximal 2-sided ideal contains a symmetric ideal (an ideal $I$ of $R$ is called symmetric if $a b c \in I$ implies $a c b \in I$ ) induces naturally a continuous mapping from $\operatorname{Max} R_{2}$ to $\operatorname{Max} R_{1}$.

\section{MAIN RESULTS}

Throughout the paper, all the rings are assumed to have identity and are not necessarily commutative. First we discuss a kind of ring which includes the class of strongly harmonic rings without using the Axiom of Choice (AC), and is the same using AC.

DEFinition 1: Let $R$ be a ring and Id $R$ the lattice of all 2-sided ideals of $R$. Then we say that Id $R$ is normal if for each pair $I_{1}, I_{2} \in \operatorname{Id} R$ with $I_{1}+I_{2}=R$, there exist $J_{1}, J_{2} \in \operatorname{Id} R$ such that

$$
I_{1}+J_{1}=R=I_{2}+J_{2} \quad \text { and } \quad J_{1} J_{2}=0 .
$$

As usual, if $R$ is a ring, then $\operatorname{Spec} R$ denotes the space of all prime 2-sided ideals of $R$ endowed with the hull-kernel topology. Define $S: \operatorname{Id} R \rightarrow \operatorname{Id} R$ by

$$
S(I)=\cap\{P \in \operatorname{Spec} R \mid I \subseteq P\}
$$


for each $I \in \operatorname{Id} R$. Then it is easy to see that the image $S(\operatorname{Id} R)$ is isomorphic to the lattice of open sets of $\operatorname{Spec} R$. Thus the topological space $\operatorname{Spec} R$ is normal if and only if for each pair $I_{1}, I_{2} \in \operatorname{Id} R$ with $S\left(I_{1}+I_{2}\right)=R$, there exist $J_{1}, J_{2} \in \operatorname{Id} R$ such that

$$
S\left(J_{1}+I_{1}\right)=R=S\left(J_{2}+I_{2}\right) \quad \text { and } \quad S\left(J_{1} J_{2}\right)=S(0) \text {. }
$$

Next, we note that if $S(I+J)=R$ and PIT holds, then $I+J=R$. In fact, if $1 \notin(I+J)$, then there is a prime ideal $P$ containing $(I+J)$ which implies that $1 \notin S(I+J)$. Hence we have:

LEMMA 1. Let $R$ be a ring and suppose PIT holds. Then $\operatorname{Spec} R$ is normal if and only if for each pair $I_{1}, I_{2} \in \operatorname{Id} R$ with $I_{1}+I_{2}=R$, there exist $J_{1}, J_{2} \in \operatorname{Id} R$ such that $I_{1}+J_{1}=R=I_{2}+J_{2}$ and $S\left(J_{1}\right) S\left(J_{2}\right) \subseteq S(0)$.

Since $S(I J)=S(I) \cap S(J) \supseteq S(I) S(J)$, we immediately have:

COROLlary. Let $R$ be a ring. If Id $R$ is normal, then $\operatorname{Spec} R$ is normal.

Theorem 1. Let $R$ be a ring and suppose PIT holds. If $\mathrm{Spec} R$ is normal, then each prime 2-sided ideal is contained in a unique maximal 2-sided ideal. Moreover, the maximal 2-sided ideal space $\operatorname{Max} R$ is a non-empty retract of $\operatorname{Spec} R$.

Proof: First we show that for each $P \in \operatorname{Spec} R$, there exists a unique maximal ideal containing it. Define

$$
F_{P}=\{I \in \operatorname{Id} R \mid I+P=R\} .
$$

Then the family $F_{P}$ satisfies

(i) If $I_{1}+I_{2} \in F_{P}$, then either $I_{1} \in F_{P}$ or $I_{2} \in F_{P}$.

(ii) If $I \in F_{P}$ and $I \subseteq J$, then $J \in F_{P}$.

In fact, if $I_{1}+I_{2} \in F_{P}$, then $I_{1}+\left(I_{2}+P\right)=R$ by definition. Then by Lemma 1 , there are $J_{1}, J_{2} \in \operatorname{Id} R$ such that $J_{1}+I_{1}=R=J_{2}+I_{2}+P$ and $S\left(J_{1}\right) S\left(J_{2}\right) \subseteq S(0)$. Moreover, we have either $J_{1} \subseteq P$ or $J_{2} \subseteq P$ since $P$ is prime and hence either $I_{1}+P=R$ or $I_{2}+P=R$, which implies either $I_{1} \in F_{P}$ or $I_{2} \in F_{P}$ by the definition of $F_{P}$. (ii) is clear.

Now let

$$
M_{P}=\sum\left\{I \in \operatorname{Id} R \mid I \notin F_{P}\right\} .
$$

We see that $1 \notin M_{P}$ by Property (i); that is, $M_{P}$ is proper. Since $P \notin F_{P}$, it follows that $P \subseteq M_{P}$. Next we show that $M_{P}$ is a maximal ideal: if $I \nsubseteq M_{P}$, then $I \in F_{P}$ and so $I+P=R$ which imlpies that $I+M_{P}=R$. Hence we have shown that for each prime 2-sided ideal of $R$, there is a maximal 2-sided ideal containing it. Note that $M_{P}$ is the unique maximal 2-sided ideal containing $P$ since $M^{\prime}=M_{M^{\prime}}=M_{P}$ for each $M^{\prime} \in \operatorname{Max} R$ with $M^{\prime} \supseteq P$. 
Now we define a mapping $m: \operatorname{Spec} R \rightarrow \operatorname{Max} R$ by sending each $P \in \operatorname{Spec} R$ to $M_{P} \in \operatorname{Max} R$. It remains to show that $m$ is continuous. Let $O_{I}$ be the set of all prime 2-sided ideals which do not contain $I$, where $I \in \operatorname{Id} R$. Then $O_{I}$ is a basic open subset of $\operatorname{Spec} R$ and it is easy to check that $O_{I}=O_{S(I)}$. Choose an $O_{I}$ with $m(P) \in O_{I} \cap \operatorname{Max} R$. Then $m(P) \nsupseteq I$ so that $I+P=R$, and hence there are $J_{1}, J_{2} \in \operatorname{Id} R$ such that $S\left(J_{1}\right) S\left(J_{2}\right) \subseteq S(0)$ and $J_{1}+I=R=J_{2}+P$; this means $J_{2} \nsubseteq P$. We claim that

$$
O_{J_{2}} \subseteq m^{-1}\left(O_{I} \cap \operatorname{Max} R\right) .
$$

In fact, if $P^{\prime} \in O_{J_{2}}$, that is, $P^{\prime} \nsupseteq S\left(J_{2}\right)$, then $P^{\prime} \supseteq S\left(J_{1}\right)$ since $S\left(J_{1}\right) S\left(J_{2}\right) \subseteq S(0)$ and $P^{\prime}$ is prime. Thus $P^{\prime}+I=R$ so that $I \in F_{P^{\prime}}$ and $I \nsubseteq m\left(P^{\prime}\right)$. Hence $m$ is continuous and this completes the proof.

COROLlary 1. Let $R$ be a ring such that Id $R$ is normal and suppose that PIT holds. Then each prime 2-sided ideal of $R$ is contained in a unique maximal 2-sided ideal.

As immediate consequences, each non-empty closed subset of Spec $R$ contains a maximal ideal and $\operatorname{Max} R$ is a non-empty compact $T_{2}$ space.

Lemma 2. Let $R$ be a ring such that $\operatorname{Id} R$ is normal. If $F$ is a closed subset of $\operatorname{Spec} R$ and $O$ is an open subset of Spec $R$ satisfying $O \supseteq(F \cap \operatorname{Max} R)$, then PIT implies $O \supseteq F$.

Proof: Suppose that $P \in F$ but $P \notin O$. Then the closure $\overline{\{P\}}$ of $P$ is disjoint from $O$. On the other hand, $\overline{\{P\}} \cap F \cap \operatorname{Max} R \neq \emptyset$ by Corollary 1 , a contradiction.

THEOREM 2. If PIT holds, then Id $R$ is normal if and only if $R$ is strongly harmonic and satisfies MIT.

Proof: Suppose that $R$ is strongly harmonic and satisfies MIT. First we shall show that for any two disjoint non-empty closed subsets $F_{1}$ and $F_{2}$ of $\operatorname{Spec} R$, there exist $J_{1}$ and $J_{2}$ in Id $R$ such that $F_{1} \subseteq O_{J_{1}}, F_{2} \subseteq O_{J_{2}}$ and $J_{1} J_{2}=0$. By Corollary 1 , we see that $\operatorname{Max} R$ is compact $T_{2}$ and that $F_{1} \cap \operatorname{Max} R$ and $F_{2} \cap \operatorname{Max} R$ are two non-empty disjoint closed subsets of $\operatorname{Max} R$ and hence are non-empty compact subsets of $\operatorname{Max} R$. Fix an $M \in F_{1} \cap \operatorname{Max} R$. Then for each $M^{\prime} \in F_{2} \cap \operatorname{Max} R$, we have $I_{M^{\prime}} \nsubseteq M^{\prime}$ and $J_{M} \nsubseteq M$ with $I_{M^{\prime}} J_{M}=0$. By the compactness of $F_{2} \cap \operatorname{Max} R$, we can find a finite number of 2-sided ideals, say $I_{1}, I_{2}, \ldots, I_{n}, J_{1}, J_{2}, \ldots, J_{n}$, such that $M \in O_{J_{1} J_{2} \ldots J_{n}}$ and $F_{2} \cap \operatorname{Max} R \subseteq O_{\left(I_{1}+I_{2}+\cdots+I_{n}\right)}$ and $I_{i} J_{i}=0,(i=1,2, \ldots, n)$. Hence

$$
\left(I_{1}+I_{2}+\cdots+I_{n}\right)\left(J_{1} J_{2} \ldots J_{n}\right)=0 .
$$

Furthermore, by Lemma 2, we have $F_{2} \subseteq O_{\left(I_{1}+I_{2}+\cdots+I_{n}\right)}$. Repeating the above procedure, we finally find $I, J \in \operatorname{Id} R$ such that $I J=0, F_{1} \subseteq O_{I}$ and $F_{2} \subseteq O_{J}$. 
Now we are going to show that Id $R$ is normal. For this, let $I_{1}, I_{2} \in \operatorname{Id} R$ with $I_{1}+I_{2}=R$ and for $i=1,2$, let

$$
D_{I_{i}}=\left\{P \in \operatorname{Spec} R \mid P \supseteq I_{i}\right\}
$$

Then $D_{I_{1}}$ and $D_{I_{2}}$ are disjoint closed subsets of Spec $R$ and so there are $J_{1}, J_{2} \in \operatorname{Id} R$ such that $O_{J_{1}} \supseteq D_{I_{1}}, O_{J_{2}} \supseteq D_{I_{2}}$ and $J_{1} J_{2}=0$. We claim that $I_{1}+J_{1}=R=I_{2}+J_{2}$. If $1 \notin I_{1}+J_{1}$, then there is a prime 2-sided ideal $P$ with $P \supseteq I_{1}+J_{1}$, which implies that $P \in D_{I_{1}}$ since $P \supseteq I_{1}$. Since $O_{J_{1}} \supseteq D_{I_{1}}$, thus $P \in O_{J_{1}}$ which means $P \nsupseteq J_{1}$, a contradiction. Hence $I_{1}+J_{1}=R$. Similarly $I_{2}+J_{2}=R$.

The other implication follows from Theorem 1. This completes the proof.

In a similar way, we obtain:

Theorem 3. (see [9, Theorem 2.3]) If PIT holds, then the lattice $\operatorname{Id}_{r} R$ of right ideals of $R$ is normal if and only if $R$ is Gelfand and satisfies MIT.

As an immediate consequence, the maximal right ideal space $\operatorname{Max}_{r} R$ of $R$ is a non-empty retract of the spectrum $\mathrm{Spec}_{\boldsymbol{T}} R$ of right ideals of $R$.

Now we study the functorial property of the assignment to each strongly harmonic ring of its maximal ideal space. Recall that an ideal $I$ of a ring $R$ is symmetric if $a b c \in I$ implies $a c b \in I$ for any $a, b, c \in R$. Then a ring $R$ is called feebly symmetric if each maximal ideal (if it exists) of $R$ contains a symmetric ideal.

Clearly a Gelfand ring is strongly harmonic, and since its maximal right ideal is symmetric, it is also feebly symmetric.

The existence of the sheaf representation of a strongly harmonic ring was established first by Koh [2] and then by Simmons [7]. We shall use the description provided by Simmons. Let $R$ be a ring. Then $I \in \operatorname{Id} R$ is called uniformly virginal if for each $a \in I$

$$
I+\operatorname{Ann}(a R)=R,
$$

where Ann $(X)$ denotes the right annihilator of $X$. We denote by $\Psi R$ the set of all such ideals. It was shown by Simmons [7, Theorem 2.4] that $\Psi R$ is a subframe of $\operatorname{Id} R$. Now for each $I \in \operatorname{Id} R$, we write Wir $(I)$ the greatest uniformly virginal ideal contained in $I$. Then

LEMma 3. [7, Theorem 5.3] Let $R$ be a ring. Then the following conditions are equivalent:

(i) $R$ is strongly harmonic.

(ii) For each $I \in \operatorname{Id} R$ and $M \in \operatorname{Max} R, \operatorname{Wir}(I) \subseteq M$ implies $I \subseteq M$.

(iii) If $I, J \in \operatorname{Id} R$ with $I+J=R$, then $\operatorname{Wir}(I)+\operatorname{Wir}(J)=R$. 
REMARK 1. We note that if $R$ is a ring with Id $R$ normal, then properties (ii) and (iii) in Lemma 3 remain true, by using only PIT.

REMARK 2. These results can be generalised to a more general setting called right unital quantales (for the details, see [10]). Also we can extend the result in Theorem 2 as follows: A ring $R$ is strongly harmonic if and only if for any two distinct maximal 2-sided ideals $M$ and $M^{\prime}$ of $R$, there exist $I_{1}, I_{2} \in \operatorname{Id} R$ such that $I_{1} \nsubseteq M_{1}, I_{2} \nsubseteq M_{2}$ and $I_{1} \cap I_{2}=0$ if and only if for any $I_{1}, I_{2} \in \operatorname{Id} R$ with $I_{1}+I_{2}=R$, there exist $J_{1}, J_{2} \in \operatorname{Id} R$ such that $I_{1}+J_{1}=R=I_{2}+J_{2}$ and $J_{1} \cap J_{2}=0$. Similar results hold for Gelfand rings.

The sheaf representation $O_{\operatorname{Max} R}$ obtained is that of which the stalk of each $M \in$ $\operatorname{Max} A$ is the factor ring $R / \mathrm{Wir}(M)$ : the canonical isomorphism from $R$ to the ring of sections is that which assigns to each $a \in R$ the section obtained by taking the canonical image $a_{M} \in R /$ Wir $(M)$ of $a \in R$, for each $M \in \operatorname{Max} R$.

DEFINITION 2: A ringed space $\left(X, O_{X}\right)$ is called quasi-local if each stalk of $O_{X}$ has a unique maximal 2-sided ideal and is called feebly symmetric if each stalk is feebly symmetric.

Following Mulvey [4] and [6], we have:

Theorem 4. Let $\left(X, O_{X}\right)$ be any ringed space and let $R$ be the ring of the global sections of $\left(X, O_{X}\right)$. If $\left(X, O_{X}\right)$ is compact and quasi-local, then Id $R$ is normal. In addition, if each stalk of $\left(X, O_{X}\right)$ is feebly symmetric, then $R$ is feebly symmetric.

Proof: The existence of maximal 2-sided ideals of $R$ follows from the fact that the ringed space $\left(X, O_{X}\right)$ is compact and quasi-local. Then suppose that $M$ and $M^{\prime}$ are distinct maximal 2-sided ideals of $R$. By the compactness theorem [5, Theorem 2.3] for ringed space, there exist $x, x^{\prime} \in X$ such that

$$
M \supseteq\left\{a \in R \mid a_{x}=0\right\} \quad \text { and } \quad M^{\prime} \supseteq\left\{a \in R \mid a_{x^{\prime}}=0\right\}
$$

Since the ringed space $\left(X, O_{X}\right)$ is quasi-local, the stalk of $O_{X}$ at each $x \in X$ has a unique maximal 2-sided ideal. Moreover, at any $x \in X$, the stalk of $O_{X}$ is isomorphic to the ring $R$ of global sections factored by the ideal $\left\{a \in R \mid a_{x}=0\right\}$. The elements $x$ and $x^{\prime}$ in $X$ corresponding to two distinct maximal ideals are therefore distinct. Since $X$ is compact and $T_{2}$, there exist two disjoint open neighbourhoods $U$ and $U^{\prime}$ of $x$ and $x^{\prime}$ in $X$, respectively. By the compactness of the ringed space $\left(X, O_{X}\right)$, there exist $a, a^{\prime} \in R$ having supports in $U$ and $U^{\prime}$ respectively, and such that $a_{x}=1$ and $a_{x^{\prime}}=1$ [5, Theorem 1.2]. Then $a \notin M$ and $a^{\prime} \notin M^{\prime}$. Furthermore, any product of elements of $R$ which contains both $a$ and $a^{\prime}$ in its expression must be zero, and so $a R a^{\prime}=0$. Hence $R$ is strongly harmonic and satisfies MIT and so Id $R$ is normal, using Theorem 2. Now, suppose in addition that $\left(X, O_{X}\right)$ is feebly symmetric and 
$M \in \operatorname{Max} R$. Then there is $x \in X$ such that $M \supseteq\left\{a \in R \mid a_{x}=0\right\}$ and hence the stalk $O_{X, x}$ is isomorphic to the ring $R$ factored by the ideal $\left\{a \in R \mid a_{x}=0\right\}$. Since each stalk is feebly symmetric, it follows that $M$ contains a symmetric ideal of $R$ so that $R$ is feebly symmetric. This completes the proof.

The converse is also true. We need the following results where we need to use Zorn's Lemma:

LEMMA 4. Let $R$ be a ring with Id $R$ normal. If $I$ is a symmetric ideal of $R$, then the minimal prime 2 -sided ideal containing $I$ is completely prime and every maximal 2-sided ideal containing $I$ contains a completely prime ideal which contains $I$.

Proof: Since $R / I$ is a symmetric ring, each minimal prime 2-sided ideal of $R / I$ is completely prime (see $[8$, Lemma 3.2$],[1]$ or $[3]$ ). The existence of minimal prime ideals is guaranteed by Zorn's Lemma.

THEOREM 5. If $R$ is a ring satisfying Id $R$ is normal, then the ringed space $\left(\operatorname{Max} R, O_{\operatorname{Max} R}\right)$ is compact and quasi-local. In addition, if $R$ is feebly symmetric, then Zorn's lemma implies that each stalk of $\left(\operatorname{Max} R, O_{\operatorname{Max} R}\right)$ is feebly symmetric.

Proof: First we note that $\operatorname{Max} R$ is a non-empty compact $T_{2}$ space. Next, for any two distinct $M_{1}$ and $M_{2}$ in $\operatorname{Max} R$, we have, by Lemma 3(iii),

$$
\operatorname{Wir}\left(M_{1}\right)+\operatorname{Wir}\left(M_{2}\right)=R \text {. }
$$

Hence there are $a_{1} \in$ Wir $\left(M_{1}\right)$ and $a_{2} \in$ Wir $\left(M_{2}\right)$ with $a_{1}+a_{2}=1$ and so $a_{2}=1-a_{1}$ is a unit in $R /$ Wir $\left(M_{1}\right)$, whence $\left(a_{2}\right)_{M_{1}}=1$. On the other hand, $\left(a_{2}\right)_{M_{2}}=0$ since $a_{2} \in \operatorname{Wir}\left(M_{2}\right)$. Hence $\left(\operatorname{Max} R, O_{\operatorname{Max} R}\right)$ is a compact ringed space. The fact that $\left(\operatorname{Max} R, O_{\operatorname{Max} R}\right)$ is a quasi-local follows from the fact that each maximal ideal of $R$ contains the ideal Wir $(M)$ for a unique $M \in \operatorname{Max} R$ by Lemma 3(ii).

Finally, if, in addition, $R$ is feebly symmetric, then for the last assertion, it suffices to show that each maximal 2-sided ideal of $R$ contains a completely prime ideal, the fact of which follows from Lemma 4 . This completes the proof.

REMARK. If $R$ is a ring satisfying the lattice $\operatorname{Id}_{r} R$ of right ideals of $R$ is normal, then PIT suffices to imply that ( $\left.\operatorname{Max} R, O_{\operatorname{Max} R}\right)$ is a compact local ringed space, in the sense of Mulvey [4].

Thus, using $\mathrm{AC}$, we then can show that the assignment to each ring $R$ of its compact quasi-local ringed space $\left(\operatorname{Max} R, O_{\operatorname{Max} R}\right)$ determines a functor from the dual of the category of strongly harmonic rings to the catogory of compact quasi-local ringed spaces. However, to show that the restriction of the above assignment to the subcategory consisting of those rings $R$ such that $\operatorname{Id}_{\mathrm{r}} R$ is normal, determines a functor, we need to use only PIT. To do this, we need one more notion. For each maximal 2-sided ideal 
$M$ (if it exists) of $R$, we write $Q_{M}$ for the union of all completely prime ideals (if any exists) of $R$ contained in $M$. Using Lemma 4, we immediately have:

Lemma 5. Let $R$ be a ring and $M$ a maximal 2-sided ideal. Then each union of symmetric ideals contained in $M$ is contained in $Q_{M}$.

Now for a given ring $R$, let $\mathrm{Qcp} R$ be the collection of all non-empty unions of completely prime ideals which is contained in some proper ideals of $R$. For $I \in \operatorname{Id} R$, we define

$$
O_{I}=\{P \in \operatorname{Qcp} R \mid I \nsubseteq P\},
$$

and consider the collection

$$
\left\{O_{I}: I \in \operatorname{Id} R\right\}
$$

to be a subbase of a topology on Qcp $R$. Then the subspace Cspec $R$ of Qcp $R$, consisting of all completely prime ideals of $R$ is precisely the subspace of Spec $R$. Moreover, we have:

Lemma 6. Let $R$ be a ring and suppose that $\operatorname{Id} R$ is normal. Then $\operatorname{Max} R$ is a retract of $Q c p R$.

Proof: Define $\mu:$ Qcp $R \rightarrow \operatorname{Max} R$ by assigning each element $Q$ of Qcp $R$, the unique maximal 2-sided ideal containing $Q$. We need to prove that $\mu$ is continuous. Let $\mathcal{F}$ be a closed subset of $\operatorname{Max} R$. Then we have to show that $\mu^{-1}(\mathcal{F})$ is closed in Qcp $R$. For this purpose, let

$$
F=\bigcap\{M \in \mathcal{F} \mid M \in \operatorname{Max} R\} \quad \text { and } \quad I=\bigcap\{Q \in \operatorname{Qcp} R \mid \mu Q \in \mathcal{F}\} .
$$

Then we observe that

$$
I=\bigcap\{P \in \operatorname{Cspec} R \mid \mu(P) \in \mathcal{F}\} .
$$

Let $Q \supseteq I$ where $Q \in \mathrm{Qcp} R$; we have to show that $\mu(Q) \in \mathcal{F}$. We first observe that if $Q$ is a prime 2 -sided ideal and

$$
Q \subseteq B=\bigcup\{M \mid M \in \mathcal{F}\}
$$

then the unique maximal 2-sided ideal containing $Q$ is in $\mathcal{F}$. In fact, we see that $Q+F$ is an ideal contained in $B$; hence there is a maximal 2-sided ideal $M$ which contains $Q+F$. Since $M \supseteq F$ and $\mathcal{F}$ is closed, thus $M \in \mathcal{F}$. Moreover, $M$ is the unique maximal ideal containing $Q$.

Now we want to find a prime 2-sided ideal $P$ which is contained in $Q \cap B$ (and hence $\mu P=\mu Q$ is in $\mathcal{F}$, as required). Consider the multiplicative system

$$
\mathcal{S}=\left\{s_{1} t_{1} s_{2} t_{2} \cdots s_{n} t_{n} \mid s_{i} \notin B, t_{i} \notin Q, i=1,2, \ldots, n, n \in N\right\} .
$$


We claim that $\mathcal{S}$ does not contain 0 . Suppose that $s_{1} t_{1} s_{2} t_{2} \cdots s_{n} t_{n}=0 \in \mathcal{S}$. Put $t=$ $t_{1} t_{2} \cdots t_{n}$. Then $t \notin Q$, since $Q \in \operatorname{Qcp} R$. Hence there exists $P^{\prime} \in \operatorname{Cspec} R \cap \mu^{-1}(\mathcal{F})$ such that $t \notin P^{\prime}$. Since $P^{\prime} \subseteq B$, it follows that each $s_{i} \notin P^{\prime}$, and since $P^{\prime}$ is a completely prime ideal, we conclude that $s_{1} s_{2} \cdots s_{n} \notin P^{\prime}$, a contradiction. Hence there exists a prime ideal $P$ disjoint from the multiplicative system, and so, in particular, $P \subseteq Q \cap B$. The continuity of $\mu$ now follows.

Lemma 7. Let $f: R_{1} \rightarrow R_{2}$ be a ring homomorphism. Then

$$
\phi=f^{-1}: Q c p R_{2} \rightarrow Q c p R_{1}
$$

is a continuous mapping.

Proof: First we note that $\phi$ sends each completely prime ideal of $R_{2}$ to a completely prime ideal of $R_{1}$, and thus sends each union of completely prime ideals of $R_{2}$ to a union of completely prime ideals of $R_{1}$. Hence $\phi$ is well-defined.

Now for each non-empty open set $O_{I}$ of Qcp $R_{1}$, we shall show that $\phi^{-1}\left(O_{I}\right)$ is open in Qcp $R_{2}$. In fact, this follows from

$$
\begin{aligned}
& \cup P_{i} \in \phi^{-1}\left(O_{I}\right) \Longleftrightarrow \phi\left(\cup P_{i}\right) \in O_{I} \\
& \Longleftrightarrow \phi\left(\cup P_{i}\right) \nsupseteq I \Longleftrightarrow f^{-1}\left(\cup P_{i}\right) \nsupseteq I \\
& \Longleftrightarrow \cup P_{i} \nsupseteq f(I) \Longleftrightarrow \cup P_{i} \in O_{f(I)}
\end{aligned}
$$

LEMma 8. The assignment $\psi$ to each maximal ideal $M$ of the union $Q_{M}$ of all completely prime ideals contained in $M$, is a continuous mapping from $\operatorname{Max} R$ to Qcp $R$.

Proof: Let $\mathcal{F}$ be a closed subset of Qcp $R$, and put

$$
F=\bigcap\{P \mid P \in \mathcal{F}\} \quad \text { and } \quad I=\bigcap\left\{M \mid Q_{M} \in \mathcal{F}\right\} .
$$

We have to show that if $M \in \operatorname{Max} R$ with $M \supseteq I$, then $Q_{M} \in \mathcal{F}$. First we see that $F \subseteq I$ and $F$ is a union of symmetric ideals since it is the intersection of unions of prime symmetric ideals. Now let $M$ be a maximal ideal containing $I$. Since $F \subseteq I$, $M$ contains $F$ so that $P_{M} \supseteq F$ by Lemma 5 and hence $P_{M} \in \mathcal{F}$ since $\mathcal{F}$ is closed. Thus the proof is completed.

Theorem 6. Let $R$ be a feebly symmetric ring satisfying $\operatorname{Id} R$ is normal. Then the mapping $\psi$ defined in Lemma 8 is a homeomorphism from $\operatorname{Max} R$ to a subspace 
of $Q_{c p} R$, consisting of those completely prime ideals with the form $Q_{M}$ for some $M \in \operatorname{Max} R$.

Proof: It is clear that $\psi$ is injective since each prime ideal is contained in a unique maximal ideal. The restriction of the mapping $\mu$, defined in Lemma 6 , is the continuous inverse of $\psi$. This completes the proof.

By Theorem 6, we can then identify $\operatorname{Max} R$ as a subspace of Qcp $R$.

Now for each ring homomorphism $f: R_{1} \rightarrow R_{2}$, define

$$
\rho_{f}=\mu f^{-1} \psi
$$

where $\mu$ and $\psi$ are as given in Lemmas 6 and 8 , respectively. The induced mapping from $\operatorname{Max} R_{2}$ to $\operatorname{Max} R_{1}$ is continuous, by Lemmas 6, 7 and 8. Therefore this determines a functor from the dual of the category of feebly symmetric rings satisfying $\operatorname{Id} R$ are normal and ring homomorphisms to the category of compact Hausdorff spaces and continuous mappings.

THEOREM 7. The assignment to each ring of the maximal ideal space Max $R$ is functorial on the category of feebly symmetric rings, with Id $R$ normal, and ring homomorphisms.

ProOF: Let

$$
R_{1} \stackrel{f}{\rightarrow} R_{2} \stackrel{g}{\rightarrow} R_{3}
$$

be ring homomorphisms. We have to show that

$$
\rho_{g f}=\rho_{f} \rho_{g}: \operatorname{Max} R_{3} \rightarrow \operatorname{Max} R_{1},
$$

or equivalently;

$$
\mu_{1} f^{-1} \psi_{2} \mu_{2} g^{-1} \psi_{3}=\mu_{1} f^{-1} g^{-1} \psi_{3}
$$

In fact, for each $M \in \operatorname{Max} R_{3}, \mu_{2} g^{-1} \psi_{3}(M)$ is the unique maximal ideal containing $g^{-1} \psi_{3}(M)$, which is a union of completely prime ideals; and $\psi_{2} \mu_{2} g^{-1} \psi_{3}(M)$ is the union of all completely prime ideals contained in $\mu_{2} g^{-1} \psi_{3}(M)$, so that

$$
\psi_{2} \mu_{2} g^{-1} \psi_{3}(M) \supseteq g^{-1} \psi_{3}(M)
$$

Hence

$$
f^{-1}\left(\psi_{2} \mu_{2} g^{-1} \psi_{3}(M)\right) \supseteq f^{-1}\left(g^{-1} \psi_{3}(M)\right) .
$$

Now the conclusion follows from the fact that each prime 2-sided ideals is contained in a unique maximal ideal. 
Definition 3: A ring homomorphism $f: R_{1} \rightarrow R_{2}$ is called fibered if for any $M_{1} \in \operatorname{Max} R_{1}$ and for any $a \in \operatorname{Wir}\left(M_{1}\right)$, we have $f(a) \in \operatorname{Wir}\left(M_{2}\right)$, for each $M_{2} \in$ $\operatorname{Max} R_{2}$ with $\rho_{f}\left(M_{2}\right)=M_{1}$.

In [5], it was shown that if $R_{1}$ and $R_{2}$ are Gelfand then each ring homomorphism is fibered. Hence we have the following generalisations of Gelfand-Mulvey duality.

THEOREM 8. (i) The functor from the category of compact quasi-local ringed spaces to the category of feebly symmetric strongly harmonic rings and fibred ring homomorphisms, obtained by assigning to each ringed space $\left(X, O_{X}\right)$ the ring of sections, determines a duality between them.

(ii) The restriction of the above functor to the subcategory consisting of those compact local ringed spaces to the category of those rings $R$ such that $\operatorname{Id}_{r} R$ is normal and ring homomorphisms, determines a duality between them and in this case we need to use only PIT.

REMARK. We still do not know whether a feebly symmetric strongly harmonic ring is Gelfand.

\section{REFERENCES}

[1] K. Koh, 'On functional representations of a ring without nilpotent elements', Canad. Math. Bull. 14 (1971), 345-352.

[2] K. Koh, 'On a representation of a strongly harmonic ring by sheaves', Pacific J. Math. 41 (1972), 459-68.

[3] J. Lambek, 'On the representation of modules by sheaves of factor modules', Canad. Math. Bull. 14 (1971), 459-466.

[4] C. Mulvey, 'A generalization of Gelfand duality', J. Algebra 56 (1979), 499-505.

[5] C. Mulvey, 'Compact ringed spaces', J. Algebra 52 (1978), 411-436.

[6] C. Mulvey, Representations of rings and modules, Lecture Notes in Mathematics 753, 1979.

[7] H. Simmons, 'Sheaf representations of strongly harmonic rings', Proc. Royal Society of Edinburgh 99A (1985), 269-275.

[8] S-H. Sun, 'Noncommutative rings in which every prime ideal is contained in a unique maximal ideal', J. Pure Appl. Algebra 76 (1991), 179-192.

[9] S-H. Sun, 'Rings in which every prime ideal is contained in a maximal right ideal', J. Pure Appl. Algebra 78 (1992), 183-194.

[10] S-H. Sun and C. Mulvey, 'Compact representations', (in preparation).

Department of Pure Mathematics

University of Sydney

New South Wales 2006

Australia 